\title{
Research on the Multiple Intelligence Theory and the Applications on Computer Music Creation
}

\author{
Sui Wu \\ Hunan City University, \\ Yiyang,Hunan,413000 China
}

\begin{abstract}
In this paper, we conduct research on the multiple intelligence theory and basic applications on the computer music creation. Music is the oldest and the most universal human and one of the art form of appeal, is a human structure to realize the thoughts and feelings through specific acoustics indispensable important form of expression and communication is an organic part of human spiritual life, it is a kind of performance art, it is an auditory art. Under this theoretical background, we use the multiple intelligence perspective as the guidance to propose the new computer music creation method that will enhance the traditional methodologies. In the future, we will combine more digital based techniques for further optimization and modification.
\end{abstract}

Keywords-Multiple Intelligence, General Applications, Computer Music, Creation, Method.

\section{Introduction}

The traditional intelligence theory is that the intelligence is an independent entity, how a person's intelligence depends largely on the genetic factors, few people can change the innate intelligence, and believe that intelligence is one unit and contemporary cognitive science and neuroscience and a lot of research achievements of anthropology fully demonstrated a fact, that is "the person's psychology and intelligence is a multifaceted, multiple elements, can't in any formal manner, using only a single pen tool properly measured". Gardner believes that each intelligence factors are equally important and should be given equal attention. Our today's education, is basically a, with emphasis on the language intelligence and mathematical logic intelligence, education, and it ignores the other intelligence [1-3].

Multiple intelligence theory is such a strong response the important reason is that it is adapt to the requirements of the times, instead of the single exam-oriented education, advocate the diverse quality education. It is, therefore, also has close relationship with aesthetic education. Because, the highlight of aesthetic education is quality education of the and it is from this angle, we believe that the theory of basic multiple intelligences study not only contributes to the development of the theory of aesthetic education in the new period, and will provide new method and theoretical weapon for the research of the aesthetic education. Accordingly, the theory has the following components. (1) The development of visual spatial intelligence. In the process of imparting knowledge and training of practical skills, the development of the students' visual spatial intelligence. In addition, with general development of virtual reality technology, to develop students' visual spatial intelligence provides a better platform. (2) The cultivation of music rhythm smart. Music rhythm intelligence development on development and the development of the entire human intelligence has the important role in migration. Teacher can make use of computer and network environment of basic music training, music appreciation, music creation. (3) The cultivation of nonverbal intelligence speech to student real-time speech recording and playback, which is beneficial to apply and develop the students' language ability, and can correct them in time in verbal language training problems [4]. 
Variety of music aesthetic appeal, especially the four relatively independent form of music culture, the differences in cultural paradigm caused the differences of aesthetic values. Out in the aesthetic strategies, four music symbols can draw lessons from and absorb each other because of the difference of aesthetic value, symbolic reference absorption will not change the aesthetic values.

The combination of computer and music as the application of digital technology in music teaching, suggests that can science and technology and art perfect combination. So far as to say that science and technology is the foundation of art, music teaching combined with modern science and technology mutual penetration, is a great opportunity to improve students' cultural quality. Different professional, different levels of students can pass it to adjust their own knowledge structure to enhance their own culture. Under normal conditions, as long as there is proper stimulation and individual's own efforts, every individual can develop and strengthen their own in any kind of basic intelligence. Affects the intellectual development of each and every one has three factors, namely, birth certificate, personal growth experience and historical and cultural background of personal survival. These three factors are mutual influence and interaction. Although people's innate aptitude to decide the type of intelligence, but the level of the intellectual development depends more on an individual historical and the cultural education activity the day after tomorrow. For the better demonstration of the corresponding methods, in the following figure one, we show the components of the multiple intelligence theory.

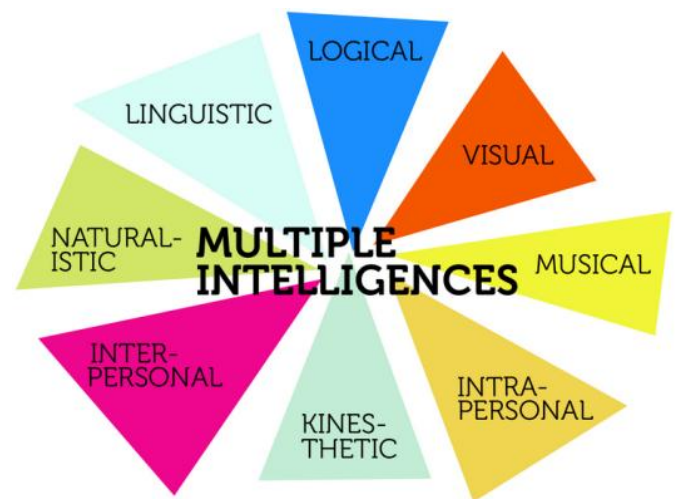

Figure 1. The Demonstration of the Multiple Intelligence Theory

In this paper, we conduct research on the multiple intelligence theory and the basic applications on the computer music creation. Compared with the traditional intelligence theory, Gardner's research puts forward a novel and practical intelligent new concept. That is: real life ability to solve practical problems facing to put forward and solve new problems to provide valuable to own culture the ability of creation and services. In the later sections, we will discuss in detail.

\section{The Proposed Methodology}

The Principles of the Musical Creation. Contracted clique composing music is in a certain special culture and background, in the fifties and sixties of the 20th century western works of music creation began to have the trend of the powerful and unconstrained style, make the ordinary people difficult to understand and accept. Under the situation to consider and study on the part of the music creators try to break this embarrassed situation, then the emergence of the general minimalist music form poses challenges to the traditional music to minimalism in produce original music criticism and opposition from many people that the music is too simple and rigid, but it reflects minimalist art in avant-garde music of academic research in the field of the larger identity [5-6].

Music is in progress is how to create tension to promote its development, and how to get help, 
that through the relaxation and achieve its overall balance, which does not include other aspects of music form research that could be reflected from the listed aspects. (1) Tones of theme and core structure of force. In music with melody line, as long as the melody line constitutes the theme or core of tones, then on the music theme and its core tone is how to play in the work force structure that is analyzed, it becomes very important and the Chinese and foreign music mostly. Music of stability and unity of the structure of the repeated may constitute the music features, and the contrast of music may constitute structure changes and turbulence, which is actually a function. Music structure, it is through these stability and instability, contrast and unity to adjust the tension and relaxation, music structure and the reasonable organization structure of music with theme and core tone plays a very important role. (2) The structure of the mode and tonality force with theme of the reasonable layout of the development mode and tonality, structure of western music is the universal writing period priority force problem. In their view, music there should be a main tone or mode. When the music is in progress from the former mode tonality, music was broken because of the balance, will show some tension, formed a kind of the tension and it actively promotes development of the music and vehicle. (3) Theme representation for traditional western music, is very important, it is the important guarantee of music embody unity and balance force. So, in a lot of music, is based on technique, at least the images of fragments of the general theme or its core tone.

The General Multiple Intelligence Theory. Harvard University psychology professor Howard Gardner's multiple intelligences theory, everyone in addition to language intelligence, mathematical logic intelligence, there are at least five other kinds of intelligence, such as music intelligence, body movement intelligence, spatial intelligence, interpersonal intelligence, intrapersonal intelligence and the naturalist intelligence. Every student has his or her advantage intelligence field to have their own learning style and methods. Multiple intelligence theory is that intelligence and certain social cultural environment evaluation standard of the people. In different social and cultural environment, people's understanding of intelligence is not the same, the requirements of the form of intelligence is not the same as this requires teachers to set up and close to real life situation, let the students to explore, and find out the ways and methods to solve the problem. Enable learners to control their own learning process that has the capacity analysis and evaluation, self-reflection and critical ability, innovative and enterprising consciousness. The figure 2 shows the principles. 


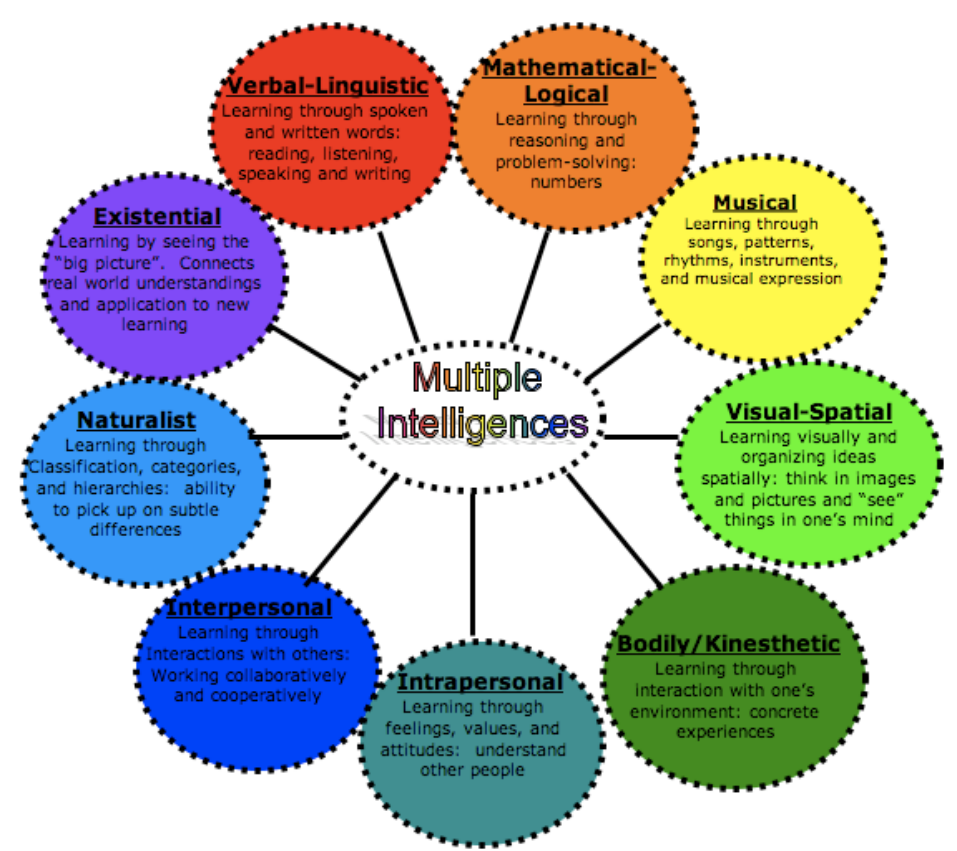

Figure 2. The Detailed Illustration of the General Multiple Intelligence Theory

The theory of basic multiple intelligences broke only based on language and mathematical logic intelligence of traditional intelligence theory understanding of intelligence, to discover and develop students with those in general traditional education has not been admitted or undiscovered intelligent strengths, facing all students, according to their aptitude, and effectively make every student get the development provides a theoretical basis [7].

Art is a deeply personal domain, the student will enter themselves and the others in the field the feelings of the world. Of course, the question of whether to taste of independent significance, if it is pure as intelligence factor that is indeed difficult to exist independently. Accordingly, Gardner of the aesthetic education and taste no independent meaning, just shows that he is still not fully get rid of the" intelligence first "theory of traditional industrial society.

The Computer Music Creation. Music of general computer cognition and intelligence creation is regarded as is one of the basic goal of the computer music, and in our country, the computer music research in the field of cognitive and intellectual creation was only the beginning. Under the support of the rapid development of the computer technology, communication technology rapid development, also the media more widely and urgent demand for basic music, traditional music are generated from artificial composition method to meet demand and wider adaptability revealed its limitations. In the past, people's music generated secret away, one of the reasons is that people overly think make music all process depends on the human level of intelligence.

Fractal music is music produced by the algorithm through multiple iterations. Self-similar fractal geometry is the essence of self-similar principle is used to construct some similar with the synthesis of small pieces of music, with minor theme in three five times return after cycle of repeat, can add some random changes in rhythm, it created the effect, whether on the macro and the micro can realistically imitate the real music. Taking the piano music as the example, we could summarize corresponding characteristics as the follows. (1) Sound realistic. The adoption of the world's most advanced digital audio sampling technology and digital to analog conversion technology, as can make play out of tone and used by the world famous concert grand piano, digital piano and has the 
characteristics of more tone. (2) Many functions. A lot of digital piano is not only beautiful timbre and tactile sensitivity as there are a lot of you unexpected features and these function exercise is very beneficial to the students. For many practitioners, rhythm instability is the biggest issue in the process of its practice and own metronome in digital piano that can fundamentally solve the problem, as there is a lot of digital piano random cabin voice recorder [8].

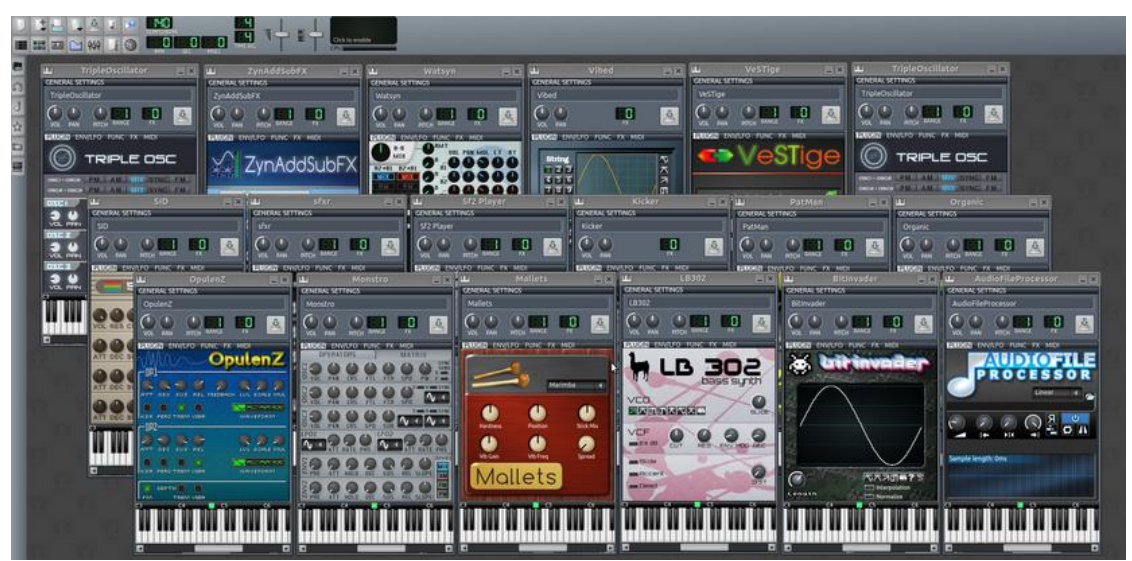

Figure 3. The Sample Computer Music Creation System

To study the fractal music broadcast to general Mandelbrot on basis of fractal sets, fractal music production technology are discussed. In front of the fractal music broadcast code first defines class of complex operation as provides various operations of the plural. Then, define a Mandelbrot music player, within the class definition tone array, and then by the random time determine the point of the complex plane, to the Mandelbrot set operation. But this program is not according to the number of iteration to determine the tone, but last time iteration, the plural value to determine the real and the imaginary part of the notes so the index value in the array to choose notes.

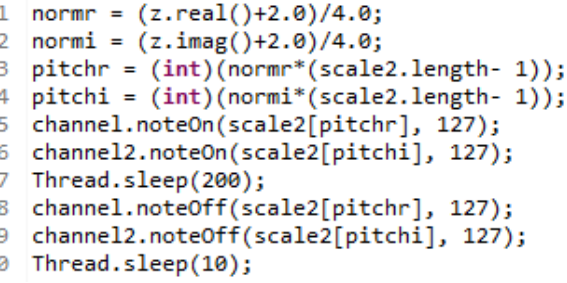

Figure 4. The Sample Code Blocks for the Proposed Coputer Music Creation Paradigm

MIDI is essentially a set of used to standardize the music equipment interconnection and general communication protocol of information exchange with each other. The significance of the emergence of MIDI is that can use the pronunciation of the modern computing technology to control the music equipment. MIDI was originally a protocol used to report the keyboard musician action that used to record a similar number keys and key speed and key, etc. Fractal image is to fractal mathematical calculated value and the color value to establish a mapping relationship and this article studies the generation of basic fractal music also is based on the fractal mathematical calculation value and note number to establish a mapping relationship to production. Mentioned algorithmic music composition in the study of mapping method is varied, and different mapping method as the music is also various.

\section{Conclusion}

In this paper, we conduct research on the multiple intelligence theory and applications on computer music creation. Composing techniques is the product of certain music culture, it has the commonness of human music culture, but also has a character of national culture, acculturation in western music culture technique specification which can't completely contain the extremely rich individual character characteristic of 
Chinese traditional music and folk music form that also cannot cover the composer's specific character, its impact is inevitable. How can mediation, conciliation, completely is the creator of value recognition, as well as the creation needs. To deal with this challenge, we propose multiple intelligence theory based music creation paradigm that will be meaningful.

\section{Reference}

[1] Takahashi, Junichi. "Multiple Intelligence Theory Can Help Promote Inclusive Education for Children with Intellectual Disabilities and Developmental Disorders: Historical Reviews of Intelligence Theory, Measurement Methods, and Suggestions for Inclusive Education." Creative Education 4.09 (2013): 605.

[2] Razmjoo, Seyyed Ayatollah, and Zahra Farmer. "On the representation of multiple intelligence types in the ILI intermediate coursebooks: A coursebook evaluation." Iranian Journal of Applied Language Studies 4.2 (2012): 153-188.

[3] Demirel, Erkan T., Muhammet Dusukcan, and Mehmet Olmez. "The impact of areas of multiple intelligence on entrepreneurial behavior." African Journal of Business Management 6.1 (2012): 415.

[4] Silver, Edward A., ed. Teaching and learning mathematical problem solving: Multiple research perspectives. Routledge, 2013.

[5] Hessler, Johannes, et al. "The MWT-B as an Estimator of Premorbid Intelligence in MCI and Dementia." Zeitschrift für Neuropsychologie (2013).

[6] Kosinski, Michal, et al. "Crowd IQ: Measuring the intelligence of crowdsourcing platforms." Proceedings of the 4th Annual ACM Web Science Conference. ACM, 2012.

[7] Peter, Mathell, et al. "Borderline personality disorder and emotional intelligence." The Journal of nervous and mental disease 201.2 (2013): 99-104.

[8] Wang, Xiaobin, Daibo Liu, and Mengshu Hou. "A novel method for multiple depot and open paths, Multiple Traveling Salesmen Problem." Applied Machine Intelligence and Informatics (SAMI), 2013 IEEE 11th International Symposium on. IEEE, 2013. 